\title{
A Preliminary Analysis on Korean University Students' Readiness Level for Industry 4.0 Revolution
}

\author{
Hasan Tinmaz ${ }^{1}$ \\ Technology Studies - Endicott College of International Studies - Woosong University - \\ Daejeon, South Korea
}

\author{
Jin Hwa Lee \\ Global Medical Services Management - Sol International School - Woosong University - \\ Daejeon, South Korea
}

\begin{tabular}{l}
\hline \hline Article history \\
\hline Received: \\
01.05 .2018
\end{tabular}

Received in revised form: 21.06.2018

Accepted:

25.06.2018

Key words: Industry 4.0; South Korea; readiness; higher education; awareness
While South Korea has been taking its place in Industry 4.0 technologies and processes (Internet of Things (IoT), Artificial Intelligence, Machine Learning, and so forth), some issues still seem untouched. One of these issues is the readiness of current university students regarding Industry 4.0 and related issues. While countries are being reshaped by Industry 4.0, people realize that some professions are vanishing, re-defined or emerging. Therefore, today's students must be ready for these Industry 4.0 changes and make their personal development agenda accordingly. Otherwise, the scenario of people losing their jobs due to technological advancements will become true. As the waves of Industry 4.0 are fast approaching to young generations of South Korean society, the study aimed to contribute to the existing Industry 4.0 literature from a social perspective. Researchers developed a literature-based survey and utilized convenient sampling approach $(\mathrm{n}=129)$ to unfold students' readiness level. Statistically analysed data explored that participants are not well aware of Industry 4.0 fundamentals and stay sceptical and alert for its implementation which is believed to bring more job opportunities whilst some current job titles disappear. It is clear that changes in higher education institutions in parallel with the emerging Industry 4.0 technologies are inevitable.

\section{Introduction}

South Korea is a well-known high-tech country in East Asia with a complicated history with regard to its neighbors; North Korea, China and Japan. According to Kemp (2017), the population of South Korea is now 51000000 with $92 \%$ of it living in cities. This demonstrates a Korean miracle when it is thought that the entire country was demolished during Korean War (1950-1953). Moreover, the Internet has infused 90\% into Korean society, where this number has stayed $50 \%$ in the World. In parallel to that infusion, social media penetration, mobile subscriptions and mobile social media use have also amplified in South Korea, especially with a comparison to the World (Table 1). 96\% percent of South Korean population states that they access the Internet for personal reasons via any device every day.

\footnotetext{
${ }^{1}$ Correspondence: htinmaz@endicott.ac.kr / hasan_tinmaz@hotmail.com
} 
Table 1. Demographics of South Korea with respect to the World

\begin{tabular}{lll}
\hline & South Korea & The World \\
\hline Total Population & 50.60 Million & 7.476 Billion \\
Urbanization & $92 \%$ & $54 \%$ \\
Internet Users & 45.49 Million & 3.773 Billion \\
Internet Penetration & $90 \%$ & $50 \%$ \\
Active Social Media Users & 41.92 Million & 2.789 Billion \\
Social Media Penetration & $83 \%$ & $37 \%$ \\
Mobile Subscriptions & 58.24 Million & 4.917 Billion \\
Mobile Subscriptions vs. Population & $115 \%$ & $66 \%$ \\
Active Mobile Social Users & 41.92 Million & 2.549 Billion \\
Mobile Social Media Penetration & $83 \%$ & $34 \%$ \\
\hline
\end{tabular}

The global average of social media penetration (monthly active accounts on the top social network in each country, compared to population) for the world is $37 \%$ and the same ratio is $83 \%$ for South Korea. The most active social media platforms in South Korea are: Youtube (72\%); Facebook (64\%); Twitter (36\%); Instagram (36\%); KakaoTalk (31\% - the Messenger); Google+ (28\%); Facebook Messenger (27\%); Line (16\%); Tumblr (13\%); LinkedIn (12\%); WeChat (10\% - Messenger in Chinese) and Twitch.TV (9\% - live streaming video platform) (Kemp, 2017).

According to the National Science \& Technology Information statistics (2016) on Technology Trade Status (An index measuring national technology transfer, used to measure the technology or the transition of the industrial structure of the country) of South Korea, there was amplification in terms of both technology import and export in 2014.

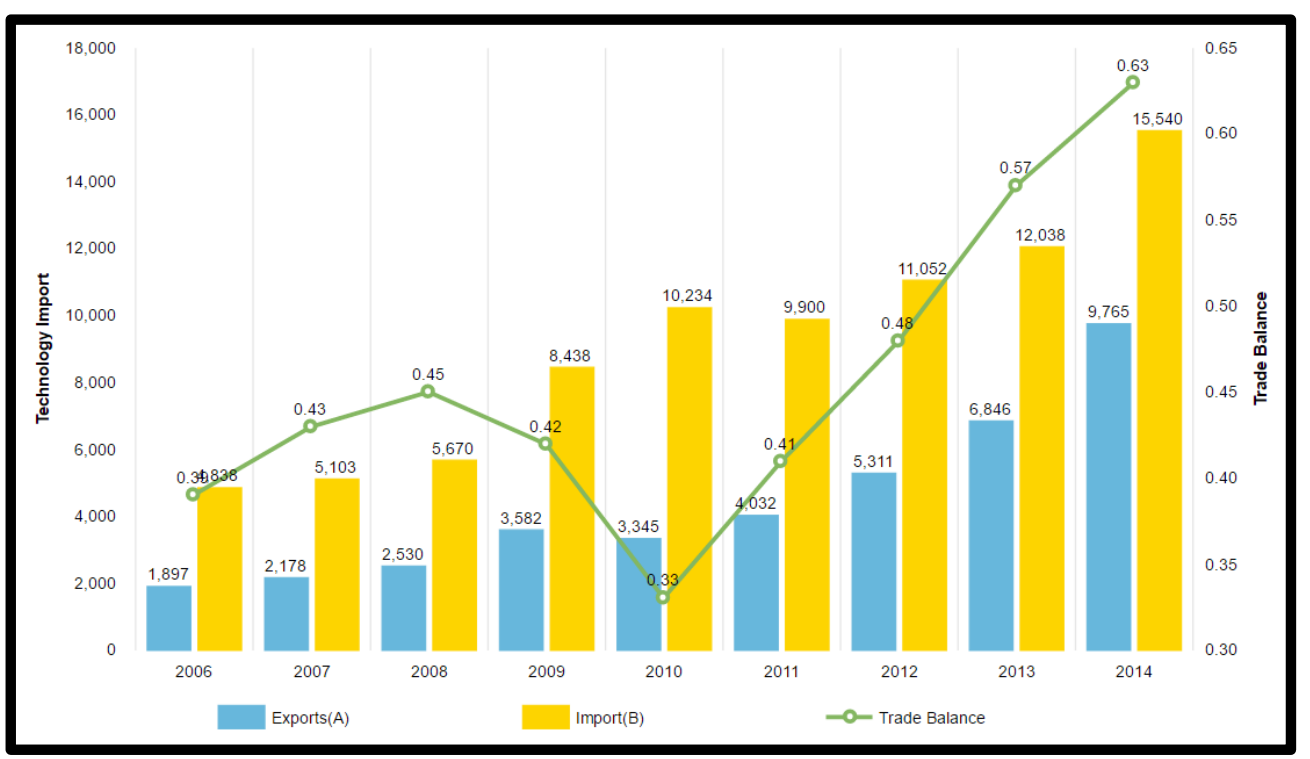

Figure 1. South Korean National Science \& Technology Information statistics

While South Korea has been keeping its place in the high-tech world, the world is faced with a revolutionary and evolutionary development in ICT (Information and Communication Technologies) which has initiated the fourth industrial revolution (Industry 4.0). It is evolutionary in a way that newer technologies have adapted previous technologies fundamentals, such as Internet of Things (IoT) stemming from existing networking tools. It is 
revolutionary that some existing technologies inherited the essence of previous developments, while they have also created newer technologies. For instance, existing networking unfolded cloud computing and big data concepts and contexts.

Traditional industries are experiencing a digital transformation which is accelerated by exponentially growing technologies (e.g. robotics, drones, sensors, 3D printing, Internet of Things (IoT), artificial intelligence (AI), big data and others) all around the world. In 2011, the German government initiated Industry 4.0 - also called as The Fourth Industrial Revolution or Industry 4.0 - acting to encourage the competitiveness of German industries. Several years after that, many other countries and industries have been implementing the processes and outcomes of Industry 4.0 to bring innovation and dynamics to their bodies. The United Nations Industrial Development Organization (UNIDO) (2017, December) defines Industry 4.0 “... as a new trend in manufacturing (and relevant sectors), based on the integration of a set of technologies that enable ecosystems of intelligent, autonomous and decentralized factories and integrated products and services" (p.5). Hence, Industry 4.0 takes manufacturing to the centre of attention while bringing recent advanced technologies to increase its quality, effectiveness and efficiency. As a result, Industry 4.0 contributes to today's manufacturing with smart machines, smart materials, and mass data as well as rapidly changing business models.

The preceding industrial revolutions unchained us from animal power, made mass production possible and opened digital doors for billions of people. This Fourth Industrial Revolution, Industry 4.0, is profoundly different: it brings together the physical, digital and biological worlds in ways we never imagined beforehand. Industry 4.0 has been a global issue in recent years and its advances in the world hold different meanings for many people depending on their age, nationality, and degree of education. However, there is a significant portion of the world population that is not aware of Industry 4.0 and upcoming changes in their lifestyle. In particular, young generations of leading countries (in terms of Industry 4.0) are currently being challenged to prepare for Industry 4.0 without proper education or specialized training in schools, colleges, and universities. It is therefore crucial to investigate Industry 4.0 readiness of current students in countries where significant changes are expected to occur due to Industry 4.0.

The readiness, as a psychological construct, has been defined as "the level of preparation for a given task sufficient to result in meaningful learning” (Psychology Dictionary, 2013). Therefore, if the universities are expecting their students to learn about Industry 4.0, they must initiate their readiness level. Moreover, the development of readiness for a professional activity is associated with the assessment of people's conditions regarding their educational background (Popov \& Puchkova, 2015). By analysing students' readiness for Industry 4.0, it will be possible to develop effective education or training courses for them. Ultimately, the positive impact of Industry 4.0 will be maximized once young generations of our society are properly trained and educated. As such, this study was conducted in a private university of South Korea where science and technology are of high value. The outcomes of this study will be beneficial not only for the leading countries of Industry 4.0 but also for other places where the influence of Industry 4.0 will reach soon.

\section{Literature Review}

Human history is experiencing a new phase; Fourth Industrial Revolution (or Industry 4.0 in short). Industry 4.0 refers to furnishing traditional industry and its processes with high 
technologies where information and communication systems (or dominantly computers) are widely utilized in the production. Industry 4.0 is related with the direct or indirect production units, which work in harmony with each other where the digital data (mostly in terms of Big Data) and software and information technologies operate in an integrated manner (Schuh, Potente, Wesch-Potente, Weber \& Prote, 2014). Industry 4.0 is striving to make computers organize and communicate among themselves autonomously without human intervention.

Industry 4.0 is currently on the rise yet; it is still unclear what Industry 4.0 brings to digital industry. However, there are three Industry 4.0 related components clearly forming digital revolution; big data, artificial intelligence and IoT (Internet of Things) based connectivity (Roblek, Mesko \& Krapez, 2016). New automation systems integrated into innovative production processes will create ways for employees to deal with the complex procedures and enable them to be more collaborative in social media platforms.

According to Roblek, Mesko and Krapez (2016), Industry 4.0 has been shaped by the development of the Internet where IoT (Internet of Things) has become the central point of fourth industrial revolution. Nowadays, billions of computers, mobile devices or other technologies are connected to each other via the Internet. Therefore, the machines communicate to each other through these connections on the Internet. As an analogy, we can say that IoT is the social media of the machines since social media, which is the social face of the Internet, has linked many people to each other using different social networks or online communities. Industry 4.0 shows similarity to social media in terms of connectivity perspective, as the ultimate aim of Industry 4.0 is to connect all devices to each other for effective communication. Industry 4.0 and the Consequences for Labour Market and Economy Report (2015) relate Industry 4.0 and social media as

Industry 4.0 stands for an interactive networking between analogue production and the digital world. This transformation includes elements such as big data, autonomously operating systems, cloud computing, social media, mobile and self-learning systems. This development is more of an evolutionary process than a revolution (p.11).

With Industry 4.0 efforts, it is intended that machines will easily adapt themselves to the smallest changes and robots will have the capacity to work in harmony with the workers by equipping the production equipment with highly automated machines and robots. Revolutions in the manufacturing sector have forced companies to keep pace with change. Just as with all industrial revolutions, in the fourth industrial revolution, production enterprises have to be able to provide competitive advantage and comply with the requirements of the times (Stock \& Seliger, 2016). New approaches, such as interoperability, decentralization and full virtualization are creating superiority for Industry 4.0 attempts (Magruk, 2016).

Magruk (2016) has also noted that the Industry 4.0 concept was born in 2011 in Germany but disseminated into many other countries. According to that new concept, software and hardware dimensions of networking will be reflected onto so-called "Smart Factories" where the processes of production will be re-shaped by leading innovative forces, such as IoT, cloud computing, big-data and others. In other words, the elements of the production processes, such as machines, humans, or even plants will be connected to each other. Even with that definition, Industry 4.0 still keeps its ambiguity in terms of its reflection on social, technological, ethical, and economical dimensions of the life. For instance; like all other revolutions in human history, Industry 4.0 revolution will bring new jobs demanding more complicated know-how and high creativity (Krawczyński, Czyżewski \& Bocian, 2016). 
Nonetheless, we still do not know what these jobs are or how youngsters will be educated for these.

Industry 4.0 is expected to bring inevitable changes in current manufacturing industry. According to a survey, $84 \%$ of German manufacturers believe that Industry 4.0 will bring a new paradigm to human labor in production (Hannover Messe Team, 2015). As mentioned by Ungeheur (2014), Industry 4.0 will bring competitive disadvantages to major production locations such as Germany due to digitization and total connectedness. In addition, small and medium-sized companies will experience more difficulties than large companies as many aspects of Industry 4.0 were not traditionally part of their value chain. On the other hand, Industry 4.0 can also bring new opportunities for manufacturing companies to develop more extensive, more efficient and more intelligent production process (Deloitte Team, 2015). Based on the survey conducted on more than 50 manufacturing companies in Switzerland by Deloitte Team (2015), more than $80 \%$ of respondents in Switzerland agreed that the digital transformation to Industry 4.0 would boost their Industry's global competitiveness and facilitate new manufacturing technologies.

In order to estimate worldwide investment for Industry 4.0, a survey was conducted on 2,000 participants from nine major industrial sectors and 26 countries (PWC, 2016). According to this survey, industrial sectors will commit US\$909 billion per annum with a major focus on digital technologies like sensors or connectivity devices, and on software and applications like manufacturing execution systems.

All these changes discussed above will also amplify security related issues. As mentioned by Sung (2017), IT security, reliability and stability needed for critical machine-to-machine communication should be carefully addressed in order to achieve successful transition toward Industry 4.0. By increasing the number and level of connections among machines and human beings, the likelihood of experiencing cyber security problems will increase. In that sense, students' readiness plays an important role to overcome and prevent cyber security related concerns. If the students are aware of cyber security challenges brought by Industry 4.0, they will create better Industry 4.0 implementation strategies in the future.

Even though there are many statistical evidences proving South Korean high-tech dominance, South Korea itself is not happy with its current condition, especially its place and attempts regarding its Industry 4.0 context. In her speech, Park Geun-hye (ex-President of South Korea) noted that "As Industry 4.0 approaches, we are unable to guarantee the future of the nation or our companies if we cannot win global competitions in securing new industries," and added that South Korea "desperately needs a future-leading transformation in science and technology, which contends for victory in creative, challenging research." (President emphasizes pioneering reforms in science, technology, 2016, September 29).

The South Korean government is not happy with its place in Industry 4.0 competition. This dissatisfaction dates back to a game between a human and a computer. The South Korean Ministry of Science, ICT and Future Planning (MSIP) Science, Technology \& ICT Newsletter number 16 (2016, April 29) starts with a result of a game between human and computer. Human beings have already lost chess matches or quiz shows to computers, which utilize AI technologies. In March 2016, another match was held between a human and computer in the traditional Chinese game called "Go" (or Baduk in Korean) in Seoul - South Korea. That "Go" match was between Lee Sedol (from South Korea), the world's number two-ranked Go player (18 times world champion), and Google DeepMind's AI program "AlphaGo". To get 
ready for the match, AlphaGo learnt about 30 million moves from 160,000 previous match records all over the world. The match result was stunning for mankind; AlphaGo: 4 - Human: 1 .

This game has captivated the attention of Korean Government to AI issues once more. According to MSIP who re-labels AI as IIT (Intelligent Information Technology), the game shows the power of ICT in cognition, learning and deduction in information processing. For MSIP, AI is one of the fundamental elements of the 4th Industrial Revolution. In that sense, South Korea must enhance its power toward AI in order to take a strong position in Industry 4.0. In the same newsletter, MSIP explains that IIT “...enables robots, automobiles and ICT devices to function on their own without human intervention, going beyond ICT industry innovation to potentially bring fundamental changes to methods of production, market structures, lifestyles and indeed all aspects of our economic and daily lives..." (p. 2). After all these attempts, South Korean MSIP announced their "Intelligent Information Industry Development Strategy" which explains major issues of AI and its integration into South Korea business and social lives.

In 2015, South Korea has initiated a project for the analysis of real-time images, called DeepView. KakaoTalk, which is a very famous messaging application in South Korea, has already been utilizing AI for keeping its users with most updated news service. Well-known South Korean companies such as Samsung and LG have set up AI groups in their structures and utilized AI in their projects (The status of artificial intelligence projects in Korea, 2016). However, South Korea started all these attempts only 2 or 3 years ago, which made the country stay behind the AI-advanced countries even though R\&D was already initiated in 2001 or earlier.

The South Korean economy is deeply rooted in China (not only recently but also historically) and the USA. Therefore, the Chinese economy's slow development and USA economy's descending global trade trends and increasing interest rates have brought many challenges to the South Korean ICT industry (Hyuk, 2015). Although South Korea has taken its place among the very high-tech countries, it has been labeled as the underdog in AI sector. South Korea has taken only $3.2 \%$ of AI world market which reached 165 billion US\$ in 2016, with an increase of $29 \%$ from 2015. To improve in AI; the South Korean government unfolded a special fund around 1 trillion Korean Won (approximately 840 million US\$) to amplify South Korea's AI industry by 2020. The Ministry of Science, ICT and Future Planning remarked that this huge fund will include funds from government and also six highly developed Korean companies; Samsung, LG, SKT, KT, Naver and Hyundai Motor where each company aims to contribute with 3 billion won (approximately 2.8 million US\$) to the total budget (Gov't to invest 1 th won in artificial intelligence, 2016, March 17). As an outcome of this attempt, on 11 October 2016, the Artificial Intelligence Research Institute (AIRI) was officially established in South Korea. The Institute will provide a research and development platform for the stakeholders of intelligence information technology (aka AI), big data and cloud computing.

South Korea has dominance on IT hardware sector; on the other hand, it seems that AI technology is not as developed as other high-tech countries. The US government planned to spend 3 billion US\$ before 2013 on an AI project called "Brain Initiative". Similarly, the EU (European Union) has approved a 1 billion euro budget for the next ten years on an AI project called "Human Brain". Korea's first significant attempt was realized in 2013 with an AI project aiming at gathering knowledge in different domains of learning and education called; 
"Exobrain Project" (The status of artificial intelligence projects in Korea (2016, March 14).

When the yearly publication of Korea Information Society Development Institute (2016); ICT Industry Outlook of Korea, was investigated for 2015 and 2016, an emphasis on Industry 4.0 as a concept was not observed. While there are references to Germany's Industry 4.0 strategies in the 2015 issue, there is no information talking about Industry 4.0 in 2016 issue. That shows a lack of interest toward Industry 4.0 among all South Korean governmental bodies.

Overall, based on the current literature review, it is evident that Industry 4.0 readiness is an urgent matter and one of the leading factors to success is to identify current readiness and prepare effective training or education towards Industry 4.0. One interesting fact is that different regions of the world seem to have different levels of awareness or readiness for Industry 4.0. For instance, a study showed that German college students had a higher awareness regarding industrial digitalization than students from Brazil (Oliveira \& Sommer, 2017). When it comes to Industry 4.0 readiness of companies, there were similarities and differences among large, medium, and small-sized companies. A study on 570 Danish companies highlighted that they possess acceptable levels of knowledge about Industry 4.0 including materials and smart production technologies, data processing, and big data (Stentoft, Rajkumar \& Madsen, 2017). However, the large-sized companies were more active and knowledgeable in terms of practicing Industry 4.0 related technologies. Regardless of their size, companies picked the topmost 3 drivers of Industry 4.0 as cost reduction, improvisation of time-to-market and legal condition. On the other hand, barriers for Industry 4.0 were revealed as lack of knowledge and lack of understanding of the strategic importance.

In order to assess Industry 4.0 readiness, there was an attempt to create a mathematical model. According to Schumacher, Erol and Sihn (2016), Industry 4.0 readiness or maturity level can be measured based on nine dimensions which include products, customers, operations, technology, strategy, leadership, governance, culture and people. Based on this model, each dimension will be assessed with the maximum score of five and it will help manufacturing companies identify their strength and weaknesses in regard to Industry 4.0 readiness.

Assessment of Industry 4.0 readiness is also evident in a study conducted by Motyl, Baronio, Uberti, Speranza and Filippi (2017). In their study, a questionnaire survey was given to 463 undergraduate students in 3 Italian universities to assess students' Industry 4.0 readiness. Based on the survey results, there was a need for effective education model to provide more structured knowledge to the students. One possible model of education was suggested by Teles dos Santos, Vianna Jr. and Le Roux (2018). This study involved chemical engineering students in Brazil with the implementation of Industry 4.0 related programming skills in their class. Students were given problems which can be solved by integrating their knowledge in chemical engineering with programming skills. Students developed computational tools to solve the problems and it became an effective training for them to reinforce Industry 4.0 readiness.

As an attempt to assess Industry 4.0 readiness of South Korean students, our study focused on the undergraduate students of a private university. As they will become the leading generation of Industry 4.0 in South Korea, findings of this study will become a valuable asset in terms of both educational and industrial perspectives. Understanding students' level of knowledge and attitude to Industry 4.0 will enable educators to implement appropriate instructional theories or models into their classrooms and effectively prepare students for future workplaces. This 
will also lead to positive outcomes in various Industry 4.0 related areas of South Korea.

\section{Method}

\section{Procedure}

The researchers searched the literature to reveal a study instrument validating the study objectives. Unfortunately, the researchers could not identify an instrument exactly fitting to the research objectives. Therefore, they developed their own survey based on Industry 4.0 literature in English. Afterwards, the draft sent to 2 content experts for validity reasons. Based on the expert feedback, a second draft of the instrument was checked by an English language expert. Due to the accessible sample size of the study, the authors cannot apply the factor analysis as being another validity feature.

As the study focuses on Korean students, the final version of the survey was translated in Korean by one of the researchers and checked by Korean language experts. At the end of these steps, the final instrument was given to 3 Korean students in order to check instrument's face validity.

Since the study aimed to obtain data regarding specific characteristics of a group (readiness toward Industry 4.0 in this study), a non-experimental survey research design was maintained. The researchers randomly selected a date (Thursday for this study), visited classes before the lunch break, and asked for the permission of the Korean students to join the study voluntarily.

Following data collection, the researchers entered the study data into SPSS for further analyses. This paper generally presents frequencies of the questions where additional mean score based tables were produced. Furthermore, Cronbach Alpha coefficient was calculated as 0.81 showing satisfactory reliability of the study. Due to lack of data sufficiency, further parametric comparisons cannot be conducted. Only gender-based comparisons were realized on Industry 4.0 perception questions. As reported by Francesconi and Parey (2018), gender gaps in university education can exist and thus it is important to assess the influence of gender gaps on students' Industry 4.0 readiness. The study conducted by Francesconi and Parey (2018) found that female students enter university with better high school grades and leave university with higher graduation rates. They utilized data from 6 cohorts of university graduates in Germany between 1989 and 2009. These data came from nationally representative longitudinal surveys by the German Center for Higher Education Research and Science Studies (DZHW).

\section{Participants}

The participants of this study $(\mathrm{n}=129)$ were undergraduate students of a private university situated in a central South Korean metropolitan city. These students were not studying in directly ICT-related disciplines. As Table 2 shows, the study participants were mostly female in gender and freshman in grade.

Table 2. Demographics of the study

\begin{tabular}{|c|c|c|c|c|c|}
\hline & \multicolumn{5}{|c|}{ Grade } \\
\hline & & Freshman & Sophomore & Junior & Total \\
\hline \multirow{2}{*}{ Gender } & Female & 47 & 26 & 17 & $90(69.8 \%)$ \\
\hline & Male & 24 & 11 & 4 & $39(30.2 \%)$ \\
\hline Total & & $71(55.0 \%)$ & 37 (28.7\%) & $21(16.3 \%)$ & $129(100 \%)$ \\
\hline
\end{tabular}




\section{Results}

After demographic questions, the participants were asked about the extent of their knowledge about the concept of "Industry 4.0". They were given the levels (Table 3) and asked to check only 1 . As Table 3 depicts, the participants mostly stated that they heard about the term but not quite sure about its real-life reflections.

Table 3. Participants' knowledge level on Industry 4.0

\begin{tabular}{llc}
\hline & $\mathrm{n}$ & $\%$ \\
\hline $\begin{array}{l}\text { I did not know "Industry 4.0" before this survey. } \\
\text { I have already heard about "Industry 4.0", but I do not understand its }\end{array}$ & 72 & 55.8 \\
applications. & & \\
I know "Industry 4.0" in general and have an idea of possible & 28 & 21.7 \\
applications. & & 3.1 \\
$\begin{array}{l}\text { I know "Industry 4.0", but I have not had any kind of related preparation } \\
\text { during my education. }\end{array}$ & 6 & 4.7 \\
I know "Industry 4.0" and I have already worked with it. & 6 & 4 \\
\hline
\end{tabular}

Subsequent to this general knowledge question, the participants were provided with a list of Industry 4.0 related technologies and processes (derived from the report of United Nations Industrial Development Organization (UNIDO), 2017) and asked to show their knowledge on each of them. 0 (zero) means "I have no knowledge on it or even I did not hear it before in my life". The other numbers from 1 (low) to 5 (high) demonstrate a certain level of knowledge on the item. Table 4 shows all the items and their frequencies. The darker background cell of each row shows the related highest frequency. Unfortunately, the most important Industry 4.0 technologies such as Internet of Things, Cloud Computing, Machine Learning, Augmented Reality, Human-Machine Interfaces and Wearable Technologies have the highest frequency for "0 (zero)". The only highest frequency with 5 score is with 3D Printing.

Table 4. The frequencies of Industry 4.0 related technologies and processes

\begin{tabular}{lllllll}
\hline \multicolumn{1}{c}{ Industry 4.0 Related Technologies and Processes } & $\mathbf{0}$ & $\mathbf{1}$ & $\mathbf{2}$ & $\mathbf{3}$ & $\mathbf{4}$ & $\mathbf{5}$ \\
\hline 1) Internet of Things (IoT) & 64 & 26 & 18 & 13 & 6 & 2 \\
2) Big Data & 28 & 27 & 23 & 31 & 14 & 6 \\
3) Cloud Computing & 44 & 28 & 27 & 14 & 8 & 8 \\
4) Machine Learning & 49 & 31 & 23 & 16 & 7 & 3 \\
5) Artificial Intelligence & 3 & 9 & 12 & 37 & 38 & 30 \\
6) Augmented Reality & 27 & 15 & 14 & 24 & 27 & 22 \\
7) Virtual Reality & 2 & 9 & 14 & 33 & 36 & 35 \\
8) Data Security, Protection and Privacy & 9 & 13 & 24 & 36 & 26 & 21 \\
9) Smart Factory & 30 & 27 & 34 & 21 & 9 & 8 \\
10) Social Web & 15 & 11 & 18 & 38 & 21 & 26 \\
11) 3D Printing & 5 & 8 & 17 & 25 & 33 & 41 \\
12) Robotics & 8 & 19 & 20 & 40 & 25 & 17 \\
13) Simulations & 6 & 11 & 15 & 39 & 29 & 29 \\
14) Human-Machine Interfaces & 36 & 20 & 23 & 33 & 10 & 7 \\
15) Smart Sensors & 7 & 15 & 19 & 44 & 26 & 18 \\
16) Wearable Technologies & 37 & 21 & 27 & 16 & 18 & 10 \\
\hline
\end{tabular}

The last question in the demographic part was how the participants define South Korea in terms of its technological development on four levels listed in Table 5. The results showed that South Korean students perceived their country as medium-high technology state. 
Table 5. Levels of South Korean perceived development

\begin{tabular}{lll}
\hline & $\mathrm{n}$ & $\%$ \\
\hline Low Technology & 2 & 1.6 \\
Medium-Low Technology & 10 & 7.8 \\
Medium-High Technology & 81 & 62.8 \\
High Technology & 36 & 27.9 \\
\hline
\end{tabular}

Subsequently, the participants were given 21 sentences referring to Industry 4.0 readiness of university students based on a Likert scale from 1 "strongly disagree" to 5 "strongly agree". Initially, the reliability analysis showed that for 21 items, Cronbach Alpha is 0.81 which is a good measure $(n=129)$. Afterwards, the mean scores and standard deviations of each item were calculated as listed in Table 6.

Table 6 shows that there is no item with a mean score higher than 4.0 (agree). A general overlook to items demonstrated that the participants moderately tend to believe in the prospective contributions of Industry 4.0 to South Korea. On the other hand, the same participants realized the fact that they were not informed about Industry 4.0 throughout their education.

Moreover, the participants consider that there will be much automation with Industry 4.0 application, which will yield to losing many regularly occupied jobs. Nonetheless, the participants are more in tendency to accept that Industry 4.0 will create different occupations that it yielded many to disappear.

The participants showed moderately less agreement with the sentence saying that South Korea is in a good position among many other countries in terms of Industry 4.0. This goes in parallel to the finding that participants see South Korea as medium-high tech country.

Table 6. Mean scores and standard deviations of survey items

\begin{tabular}{lll}
\hline Survey Questions & M & SD \\
\hline 1. Industry 4.0 is a topic of discussion in my department. & 2.55 & 0.97 \\
2. I will be dealing with aspects of Industry 4.0 more and more in the future & 3.84 & 0.89 \\
in my working environment. & & \\
3. I have a clear idea of how Industry 4.0 will change my future working & 3.28 & 0.68 \\
environment. & 3.98 & 0.74 \\
4. Industry 4.0 will create permanent changes in the way people work. & 2.60 & 1.01 \\
5. I am sufficiently informed about Industry 4.0 by my department. & 3.26 & 0.87 \\
6. Korea is in a good position among many other countries in terms of & & \\
Industry 4.0. & 3.63 & 0.92 \\
7. I have a strategy to develop my Industry 4.0 skills and knowledge. & 3.34 & 0.84 \\
8. I think there is a lack of knowledge about Industry 4.0 in Korea. & 3.42 & 0.84 \\
9. I think there is a lack of understanding of the strategic importance of & & \\
Industry 4.0 & 3.40 & 0.79 \\
10. I have enough motivation to work with Industry 4.0 & 3.29 & 0.78 \\
11. I am competent enough to work with Industry 4.0 & 3.00 & 0.79 \\
12. I have adequate knowledge about Industry 4.0 to evaluate its importance. & 3.43 & 0.73 \\
13. Industry 4.0 must be a top priority in Korea. & 3.60 & 0.89 \\
14. People will lose their jobs because of Industry 4.0. & 3.40 & 0.79 \\
15. I think there is a lack of employee readiness for Industry 4.0. & \\
& Participatory Educational Research (PER) \\
\end{tabular}




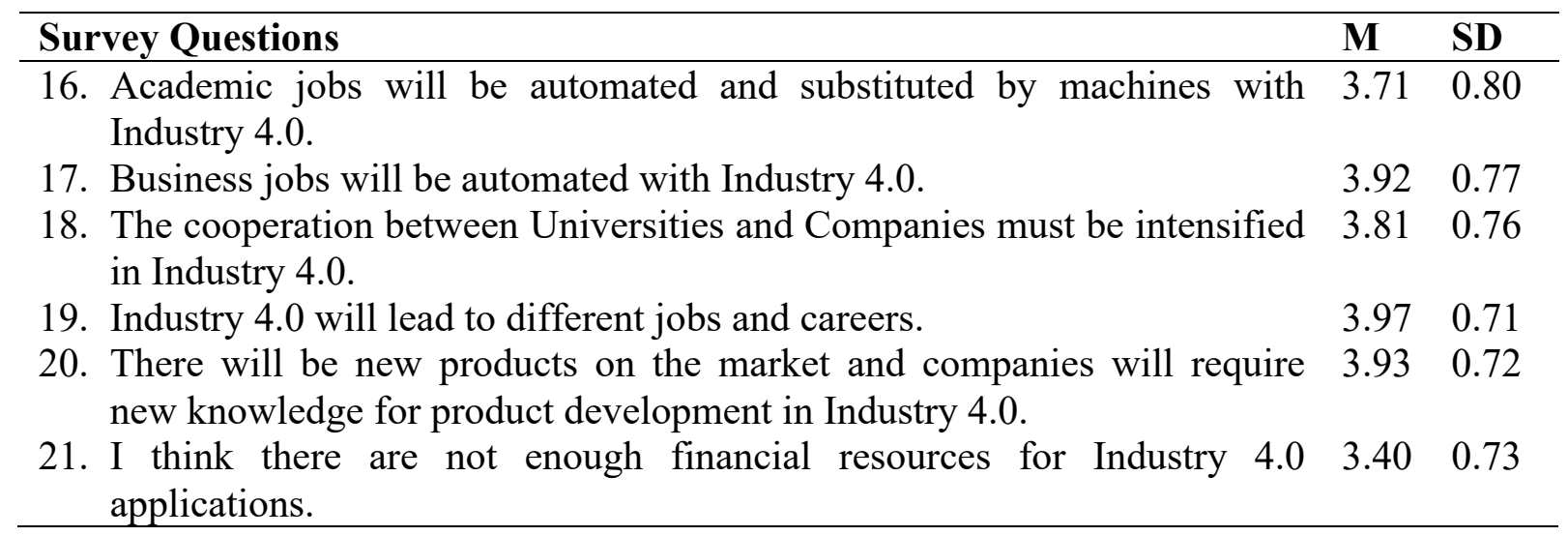

Lastly, the same 21 questions were tested whether or not there is a significant statistical difference according to gender variable. Independent samples t-tests showed that there are only 5 items differing in relation to gender variable in the survey. For all five items, males scored more than female participants. Therefore, it seems that male participants tend to believe in the superiority of South Korea for Industry 4.0 and are more motivated and competent toward working within Industry 4.0 context (Table 7 ).

Table 7. Independent sample t-test results according to gender variable

\begin{tabular}{lllllll}
\hline Factor & Gender & $\mathrm{n}$ & $\mathrm{M}$ & $\mathrm{SD}$ & $t$ & $p$ \\
\hline \multirow{2}{*}{ Question 6 } & Female & 90 & 3.16 & 0.81 & -2.055 & .044 \\
& Male & 39 & 3.51 & 0.94 & & \\
Question 10 & Female & 90 & 3.21 & 0.74 & -4.525 & .000 \\
\multirow{2}{*}{ Question 11 } & Male & 39 & 3.85 & 0.70 & & \\
& Female & 90 & 3.13 & 0.72 & -3.719 & .000 \\
Question 13 & Male & 39 & 3.67 & 0.80 & & \\
\multirow{2}{*}{ Question 18 } & Female & 90 & 3.32 & 0.71 & -2.720 & .007 \\
& Male & 39 & 3.69 & 0.69 & & \multirow{2}{*}{009} \\
\hline
\end{tabular}

\section{Discussion and Conclusion}

According to the results of this study, a significant number of students among the sample were either planning to work overseas or unsure of their future job locations. This reflects uncertainty or unpredictability of the changes that Industry 4.0 will bring to the young generation of South Korean society. Although Industry 4.0 is a commonly spoken term that most of the students are aware of, a deeper understanding of its concept and involvement with any related activity were not evident in this study. This could be due to a lack of education or adequate training in higher education institutions of South Korea as the key concepts of Industry 4.0 were not yet incorporated into educational curriculum. As evident in a previous study (Oliveira \& Sommer, 2017), Industry 4.0 awareness and possession of related knowledge can produce different behavioral outcomes from the students. As such, a future study can be carried out in the form of a comparative analysis on students' attitudes towards future career paths before and after Industry 4.0 related education or training.

In this study, students chose Internet of Things as the most unfamiliar technology whereas 3D printing was the most well-known. It signifies a lack of Industry 4.0 readiness among the young generation of South Korea as Internet of Things is one of the most fundamental 
technologies for Industry 4.0. Internet of Things is defined as IT systems connected to all subsystems, processes, internal and external objects, suppliers, and customer networks that communicate and cooperate with each other and with humans (Smit, Kreutzer, Moeller \& Carlberg, 2016). Yet this recent innovative approach was not properly introduced to the students and, without immediate actions, it may cause a significant delay for Industry 4.0 readiness in South Korea. One of the possible solutions will be running more government funded projects in higher education institutions with a major focus on unfamiliar Industry 4.0 technologies. A student-centered collaboration between university and Industry can also be a possible solution as suggested by Ruggiero (2015).

Although students may possess limited knowledge about Industry 4.0, they seem to believe it will bring many changes to South Korea. This could be due to the fact that students view their country as a medium-high technology state and thus more exposed to Industry 4.0. Nevertheless, these upcoming changes can bring both positive and negative influences on future career paths. Due to this uncertainty, students' response to survey questions in general may have been more or less moderate. However, this is not only the case for students in South Korea. A survey on 302 companies in Europe showed similar responses in terms of the uncertainty that Industry 4.0 will bring (Expense Reduction Analysts, 2015). More than half of the participants had no clear idea of what is behind the notion of Industry 4.0. Even companies for which digitalization is of great importance had, with almost $50 \%$ of all businesses surveyed, no clear idea of what Industry 4.0 exactly is.

To some extent, students viewed Industry 4.0 in different perspectives depending on their gender. One possible explanation might be that male students are more knowledgeable and more interested in Industry 4.0 technologies or they could have been involved in Industry 4.0 related projects more often. It was previously reported that Industry 4.0 experts view the upcoming changes in a different way from non-experts (Expense Reduction Analysts, 2015). Therefore, future study will be required to clarify the relationship between gender difference and Industry 4.0 related knowledge.

Last but not least, it is important to focus on the study limitations. This study was conducted with a specific group of students. Hence, study results are highly dependent on this context. Therefore, the same study should be replicated within other contexts in order to validate the findings. This also includes the factor analysis of the instrument with a larger sample. Moreover, this limited sample of university students is not necessarily representative of all other university students in South Korea. Besides, the following limitations are relevant to the study: (a) the validity of this study is limited to the reliability of the instruments, and (b) validity is limited to the honesty of the participants' responses to the study instruments.

\section{References}

Deloitte Team (2015). Industry 4.0 Challenges and solutions for the digital transformation and use of exponential technologies. Retrieved from https://www2.deloitte.com/content/dam/Deloitte/ch/Documents/manufacturing/ch-enmanufacturing-industry-4-0-24102014.pdf

Expense Reduction Analysts (2015). Vision for the future: Are you ready for Industry 4.0? Retrieved from http://cz.expensereduction.com/fileadmin/user_upload/en/OTHERS_EN/study_vision _for_the_future_2015.pdf 
Francesconi, M. \& Parey, M. (2018). Early Gender Gaps among University Graduates. European Economic Review.

Gov't to invest 1 tln won in artificial intelligence (2016, March 17). YonHap News Agency. Retrieved from http://english.yonhapnews.co.kr/news/2016/03/17/0200000000AEN20160317003751 320.html

Hannover Messe Team (2015). How far are we? Industry 4.0. Retrieved from http://www.messe.de/home

Hyuk, C. (2015). ICT industry long-term forecast (2016-2020) and strategy. Policy Research, 15(4), 1-118.

Industry 4.0 and the Consequences for Labour Market and Economy Report (2015). Institute for employment research. Retrieved from http://doku.iab.de/forschungsbericht/2015/fb0815 en.pdf

Kemp, S. (2017). Digital, social \& mobile in 2016: We are social's compendium of global digital statistics. Retrieved from http://wearesocial.com/

Korea Information Society Development Institute (2016). ICT industry outlook of Korea. Retrieved from http://www.kisdi.re.kr/kisdi/jsp/fp/kr/main.jsp

Krawczyński, M., Czyżewski, P., \& Bocian, K. (2016). Reindustrialization: A challenge to the economy in the first quarter of the twenty-first century. Foundations of Management, 8, 107-122.

Magruk, A. (2016). Uncertainty in the sphere of the Industry 4.0 - potential areas to research. Business, Management and Education, 14(2), 275-291.

Motyl, B., Baronio, G., Uberti, S., Speranza, D. \& Filippi, S. (2017). How will change the future engineers' skills in the Industry 4.0 framework? A questionnaire survey. Procedia Manufacturing, 11, 1501-1509.

National Science \& Technology Information Statistics (2016). Technology trade status. Retrieved from http://www.ntis.go.kr/en/GpTechnologyTradeStatus.do

Oliveira, P.S. \& Sommer, L. (2017). Globalization and digitalization as challenges for a professional career in manufacturing industries - Differences in awareness and knowledge of students from Brazil and Germany. Education Sciences, 7(55), 1-13.

Popov, L. M. \& Puchkova, I. M. (2015). The model of the psychological readiness of students to the professional activity: Theory and practice. The Social Science, 10(5), 646-650.

President emphasizes pioneering reforms in science, technology (2016, September 29). The Korean culture and information service. Retrieved from http://www.korea.net/NewsFocus/policies/view?articleId=141098

Psychology Dictionary (2013). Readiness. Retrieved from https://psychologydictionary.org/readiness/

PWC (2016). Industry 4.0: Building the digital enterprise. Global Industry 4.0 survey. Retrieved from http://www.pwc.com/Industry40

Roblek, V., Mesko, M. \& Krapez, A. (2016). A complex view of Industry 4.0. Sage Open, April-June, 1-11.

Ruggiero, M.E. (2015). University and industry: an experience in teaching, research and artistic expression. Social and Behavioral Sciences, 174, 4038-4045.

Schuh, G., Potente, T., Wesch-Potente, C., Weber, A. R., \& Prote, J. P. (2014). Collaboration mechanisms to increase productivity in the content of Industrie 4.0. Paper presented at Robust Manufacturing Conference (RoMaC 2014): Bremen, Germany. Elsevier Science. https://doi.org/10.1016/j.procir.2014.05.016

Schumacher, A., Erol, S. \& Sihn, W. (2016). A maturity model for assessing Industry 4.0 readiness and maturity of manufacturing enterprises. Procedia CIRP, 52, 161-166. 
Science, Technology \& ICT Newsletter 16 (2016, April 29). MSIP [Ministry of Science, ICT and Future Planning] announces development strategy for the intelligence information industry.

Retrieved

from

http://english.msip.go.kr/english/msipContents/contentsView.do?cateId=msse44\&artI $\mathrm{d}=1296203$

Smit, J., Kreutzer, S., Moeller, C. \& Carlberg, M. (2016). Industry 4.0. Policy department A: Economic and scientific policy. European Parliament. Retrieved from http://www.europarl.europa.eu/RegData/etudes/STUD/2016/570007/IPOL_STU(2016 )570007_EN.pdf

Stentoft, J., Rajkumar, C., \& Madsen, E.S. (2017). Industry 4.0 in Danish Industry: An empirical investigation of the degree of knowledge, perceived relevance and current practice. Retrieved from http://www.efnms.eu/wp-content/uploads/2017/06/StentoftRajkumar-Madsen-2017-Industry_4_0_in_Danish_Industry.pdf

Stock, T., \& Seliger, G. (2016). Opportunities of Sustainable Manufacturing in Industry 4.0. Paper Presented at 13th Global Conference on Sustainable Manufacturing Decoupling Growth from Resource Use: Ho Chi Minh City, Vietnam. Elsevier Science. https://doi.org/10.1016/j.procir.2016.01.129

Sung, T.K. (2017). Industry 4.0: A Korea perspective. Technological Forecasting \& Social Change.

Teles dos Santos, M., Vianna Jr., A.S., \& Le Roux, G.A.C. (2018). Programming skills in the industry 4.0: are chemical engineering students able to face new problems?. Education for Chemical Engineers, 22, 69-76.

The status of artificial intelligence projects in Korea (2016, March 14). KBS World. Retrieved from

http://world.kbs.co.kr/english/program/program_economyplus_detail.htm? $\mathrm{No}=5420$

Ungeheur, I. U. (2014, November). Personal Interview. Information technology security will make or break Industry 4.0. Retrieved from http://www.oliverwyman.com/content/dam/oliver-wyman/global/en/2014/nov/14-128ow_pers_en_07.pdf

United Nations Industrial Development Organization (UNIDO). (2017, December). Industry 4.0: Opportunities behind the challenge - background paper. Retrieved from https://www.unido.org/sites/default/files/files/2017-

11/UNIDO\%20Background\%20Paper\%20on\%20Industry\%204.0_27112017.pdf 